\title{
Recurrent Processes Emulate a Cascade of Hierarchical Decisions: Evidence from Spatio-Temporal Decoding of Human Brain Activity.
}

\author{
Laura Gwilliams ${ }^{1,2} \quad$ Jean-Remi King ${ }^{1,3,4,5}$ \\ ${ }^{1}$ Department of Psychology, New York University, ${ }^{2}$ NYU Abu Dhabi Institute, ${ }^{3}$ Frankfurt Institute for Advanced \\ Studies, ${ }^{4}$ Ecole Normale Supérieure, ${ }^{5} \mathrm{CNRS}$
}

\begin{abstract}
Mounting evidence suggests that perception depends on a largely-feedforward brain network. However, the discrepancy between (i) the latency of the corresponding feedforward responses (150-200 ms) and (ii) the time it takes human subjects to recognize brief images (often $>500 \mathrm{~ms}$ ) suggests that recurrent neuronal activity is critical to visual processing. Here, we use magneto-encephalography to localize, track and decode the feedforward and recurrent responses elicited by brief presentations of variably-ambiguous letters and digits. We first confirm that these stimuli trigger, within the first $200 \mathrm{~ms}$, a feedforward response in the ventral and dorsal cortical pathways. The subsequent activity is distributed across temporal, parietal and prefrontal cortices and leads to a slow and incremental cascade of representations culminating in action-specific motor signals. We introduce an analytical framework to show that these brain responses are best accounted for by a hierarchy of recurrent neural assemblies. An accumulation of computational delays across specific processing stages explains subjects' reaction times. Finally, the slow convergence of neural representations towards perceptual categories is quickly followed by all-or-none motor decision signals. Together, these results show how recurrent processes generate, over extended time periods, a cascade of hierarchical decisions that ultimately predicts subjects' perceptual reports.
\end{abstract}

Keywords: Perceptual decision making, Magneto-encephalography, Recurrent processes

\section{Introduction}

To process the rich sensory flow emanating from the retina, the brain recruits a hierarchical network originating in the primary visual areas and culminating in the infero-temporal, dorso-parietal and prefrontal cortices. $[1,2,3,4]$.

In theory, the feedforward recruitment of this neural hierarchy could suffice to explain our ability to recognize visual objects. For example, recent studies demonstrate that artificial feedforward neural networks trained to categorize objects generate similar activations patterns to those elicited in the infero-temporal cortices $[5,6]$.

However, feedforward architectures have a fixed number of processing stages, and are thus unable to explain a number of neural and perceptual phenomena. For example, the time it takes subjects to recognize objects considerably varies from one trial to the next [7]. In addition, the neural responses to visual stimuli generally exceed the $200 \mathrm{~ms}$ feedforward recruitment of the visual hierarchy $[8,9]$.

A large body of research shows that recurrent processing accounts for such behavioral and neural dynamics $[9,10,11,12,13,14]$. In this view, recurrent processing would mainly consist in accumulating sensory evidence until a decision to act is triggered [13].

However, the precise neuronal and computational organization of recurrent processing remains unclear at the system level. In particular, how distinct recurrent assemblies implement series of hierarchical decisions remains a major unknown.

To address this issue, we use magnetoencephalography (MEG) and structural magneticresonance imaging (MRI) to localize, track and decode, from whole-brain activity, the feedforward (0-200ms) and recurrent processes $(>200 \mathrm{~ms}$ ) elicited by variably ambiguous characters briefly flashed on a computer screen. We show that the late and sustained neural activity distributed along the visual pathways generates, over extended time periods, a cascade of categorical 

F). decisions that ultimately predicts subjects' perceptual 90 reports.

\section{Results}

\subsection{Subjective reports of stimulus identity are categor ical}

To investigate the brain and computational bases of perceptual recognition, we used visual characters as described in [15]. These stimuli can be parametrically 99 morphed between specific letters and digits by varying 100 the contrast of their individual edges, hereafter referred 101 to as pixels (Fig.1A-B).

To check that these stimuli create categorical per- 103 cepts, we asked eight human subjects to provide contin- ${ }^{104}$ uous subjective reports by clicking on a disk after each 105 stimulus presentation (Experiment 1. Fig.1A). The ra- 106 dius and the angle of the response on this disk indicated 107 the subjective visibility and the subjective identity of 108 the stimulus respectively. We then compared (i) the re- 109 ported angle with (ii) the stimulus evidence (i.e. the ex- 110 pected angle given the pixels) for each morph separately 111 (e.g. 5-6, 6-8, etc). Subjective reports were categori- 112 cal: cross-validated sigmoidal models better predicted ${ }_{113}$ subjects' responses $(\mathrm{r}=0.49+/-0.05, \mathrm{p}=0.002)$ than lin- ${ }_{114}$ ear models $(\mathrm{r}=0.46+/-0.043, \mathrm{p}=0.002$, sigmoid $>$ linear: 115 $\mathrm{p}=0.017$ Fig. $1 \mathrm{~B}-\mathrm{C})$.

We adapted this experimental paradigm for an MEG ${ }_{117}$ experiment by modifying three main aspects (Experi- 118 ment 2). First, we used stimuli that could be morphed ${ }_{119}$ between letters and digits, to trigger macroscopically 120 distinguishable brain responses in the visual word form area (VWFA) and number form area (NFA) [16, 17]. 122 Second, we added two task-irrelevant flankers next to 123 the target stimulus (Fig.1D) to increase our chances of 124 eliciting recurrent processes via crowding [18]. Third, a 125 new set of seventeen subjects reported subjective iden- 126 tity via a two-alternative forced-choice button press. ${ }_{127}$ The identity-response mapping was orthogonal to the 128 letter/digit category and changed on every block of $48 \quad 129$ trials. There were 1920 trials total, 320 of which were ${ }_{130}$ presented passively and did not require a response. $\quad 131$

Perceptual reports followed a similar sigmoidal pat- ${ }_{132}$ tern to Experiment 1: performance was worse for 133 more ambiguous trials $(65 \%)$ as compared to unam- 134 biguous trials $(92 \%, \mathrm{p}<0.001)$. In addition, reaction 135 time slightly, and consistently, increased with difficulty. ${ }_{136}$ For example, highly ambiguous stimuli were identified ${ }_{137}$ within $690 \mathrm{~ms}$, whereas nonambiguous stimuli were ${ }_{138}$ identified within $624 \mathrm{~ms}\left(\mathrm{z}=-21.68, \mathrm{p}<0.001\right.$ (Fig.1E- ${ }_{139}$

\subsection{Neural representations are functionally organized over time and space}

Here, we aimed to decompose the sequence of decisions that allow subjects to transform raw visual input into perceptual reports. To this aim, we localized the MEG signals onto subjects' structural MRI with dynamic statistical parametric mapping (dSPM, [19]), and morphed these source estimates onto a common brain coordinate [20,21]. The results confirmed that the stimuli elicited, on average, a sharp response in the primary visual areas around $70 \mathrm{~ms}$, followed by a fast feedforward response along the ventral and dorsal visual pathways within the first 150-200 ms. After $200 \mathrm{~ms}$, the activity appeared sustained and widely distributed across the associative cortices up until 500-600 ms after stimulus onset (Fig.1G and Supplementary Video 1).

To separate the processing stages underlying these neural responses, we applied i) mass-univariate; ii) temporal decoding and iii) spatial decoding analyses based on the five orthogonal features varying in our study: (1) the position of the stimulus, (2) its identity, (3) its perceived category, (4) its difficulty and (5) its corresponding button press.

First, we aimed to identify when and where low-level visual features would be represented in brain activity. To do so, we estimated, at each time sample separately, the ability of an 12-regularized logistic regression to predict, from all MEG sensors, the position of the stimulus on the computer screen (left versus right). Stimulus position was decodable between 41 and 1,500 $\mathrm{ms}$ and peaked at $120 \mathrm{~ms}(\mathrm{AUC}=0.94 ; \mathrm{SEM}=0.007 ; \mathrm{p}<0.001$ as estimated with second-level non-parametric temporal cluster test across subjects, (Fig.2C). These signals peaked in the early visual cortex (mean MNI ( $\mathrm{x}=27.59$; $y=-74.15 ; z=-1.07)$ ), and propagated along the ventral and dorsal streams during the first $200 \mathrm{~ms}$ (Fig.2A, supplementary video). To summarize where stimulus position was represented in the brain, we implemented 'spatial decoders': 12-regularized logistic regressions fit across all time samples $(0-1,500 \mathrm{~ms})$ for each estimated brain source separately. Spatial decoding peaked in early visual areas and was significant across a large variety of visual and associative cortices as estimated with a second-level non-parametric spatial cluster test across subjects (Fig.2B), confirming the retinotopic organization of the visual hierarchy $[22,23]$.

Second, we aimed to isolate more abstract representations related to stimulus identity. Stimulus identity can be analyzed either from an objective referential (what stimulus is objectively presented?) or from a subjective referential (i.e. what stimulus did subjects report 


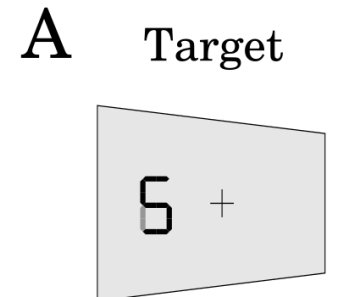

$83 \mathrm{~ms}$

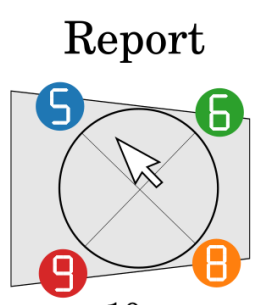

$10 \mathrm{~s}$
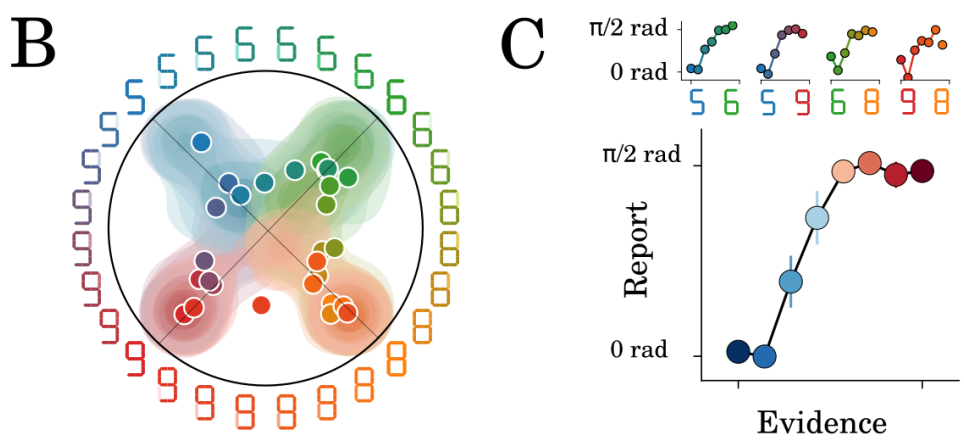

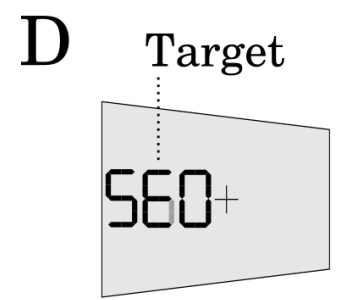

$100 \mathrm{~ms}$

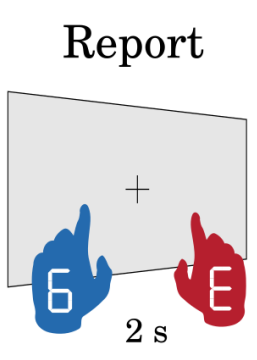

$\mathrm{s}$

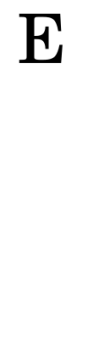

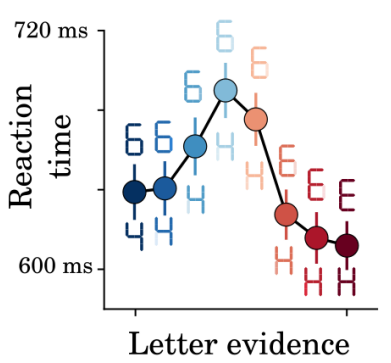

F

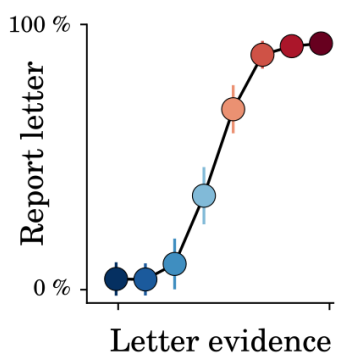

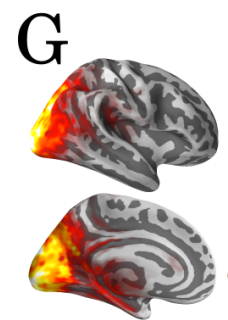

100

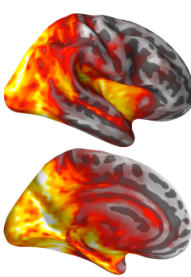

200

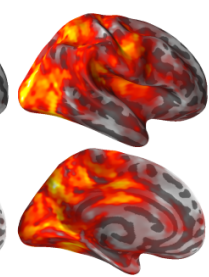

300

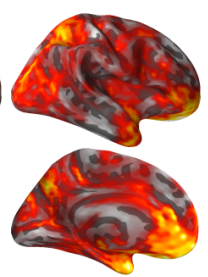

400

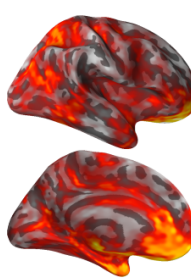

500

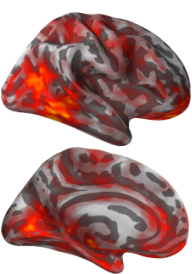

600

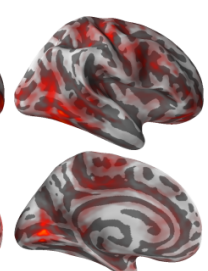

700

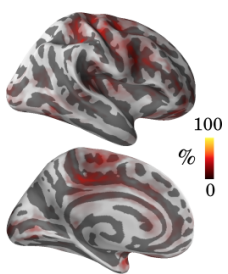

$800 \mathrm{~ms}$

Figure 1: Experimental protocol and behavioral results. Experiment 1: 8 human subjects provided perceptual judgments on variably ambiguous digits briefly flashed at the center of a computer screen (A). Reports were made by clicking on a disk, where (i) the radius and (ii) the angle on the disk indicate (i) subjective visibility and (ii) subjective identity respectively. (B) Distribution (areas) and mean response (dots) location for each color-coded stimulus. (C) Top plots show the same data as B, broken down for each morph set. The X-axis indicates the expected angle given the stimulus pixels (color-coded), hereafter referred to as evidence. The y-axis indicates the angle of the mean response relative to stimulus evidence. The bottom plot shows the same data, grouped across morphs. (D) Experiment 2: 17 subjects categorized a briefly flashed and parametrically manipulated-morph using a two-alternative forced-choice. Stimulus-response mapping changed on every block. (E) Mean reaction times as a function of categorical evidence (the extent to which the stimulus objectively corresponds to a letter). (F) Mean probability of reporting a letter as a function of categorical evidence. (G) Evoked activity estimated with dSPM and estimated across all trials and all subjects. These data are also displayed in Supplementary Video 1. Error-bars indicate the standard-error-of-the-mean (SEM) across subjects.

having seen?). We first focus on decoding features of ${ }_{152}$ the stimulus that are not ambiguous, such that subjec- ${ }_{153}$ tive and objective representations are confounded. To this aim, we grouped stimuli along common continua ${ }^{154}$ (e.g. The eight stimuli along the 4-H continuum belong ${ }^{155}$ to the same morph and are here considered to share a ${ }^{156}$ common identity) and fit logistic regression classifiers ${ }^{157}$ across morphs (i.e. E-6 versus 4-H). The correspond- 158 ing stimulus identity was decodable between 120 and 159 $845 \mathrm{~ms}$ and peaked at $225 \mathrm{~ms}(\mathrm{AUC}=0.59 ; \mathrm{SEM}=0.01 ; 160$ $\mathrm{p}<0.001)$. These effects peaked more anteriorly than ${ }^{161}$ those of stimulus position (mean MNI: $x=27.75 ; y=-$ $62.75 ; z=-1.55 ; p<0.001)$.

Third, we aimed to isolate the neural signatures of subjective perceptual categorization and thus focus on decoding ambiguous pixels. To this aim, we grouped stimuli based on whether the subject reported a digit or to a letter category. Temporal decoders weakly but significantly classified perceptual category from 150 to $940 \mathrm{~ms}$ after stimulus onset and peaked at $370 \mathrm{~ms}$ (AUC $=55 \%$; SEM $=0.01 ; \mathrm{p}<0.001$, Fig. $2 \mathrm{C}$ ). The corresponding sources also peaked in the inferotemporal cor- 
bioRxiv preprint doi: https://doi.org/10.1101/840074; this version posted November 12, 2019. The copyright holder for this preprint (which was not certified by peer review) is the author/funder, who has granted bioRxiv a license to display the preprint in perpetuity. It is made available under aCC-BY-NC-ND 4.0 International license.
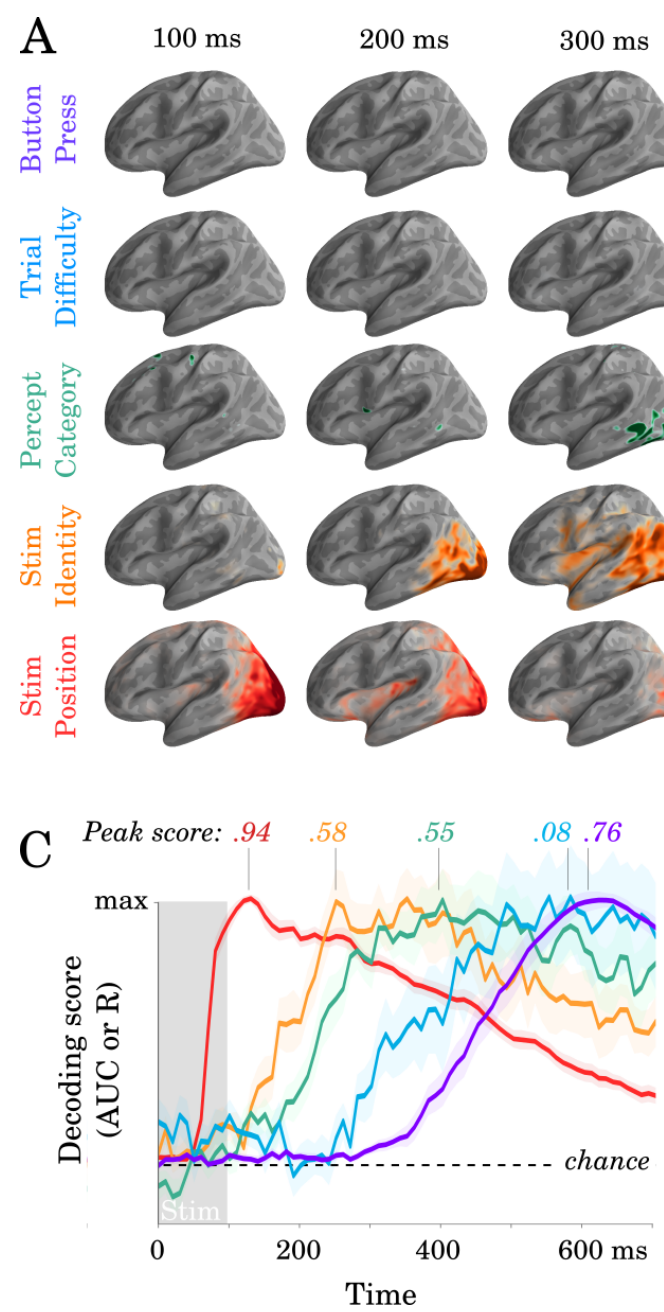
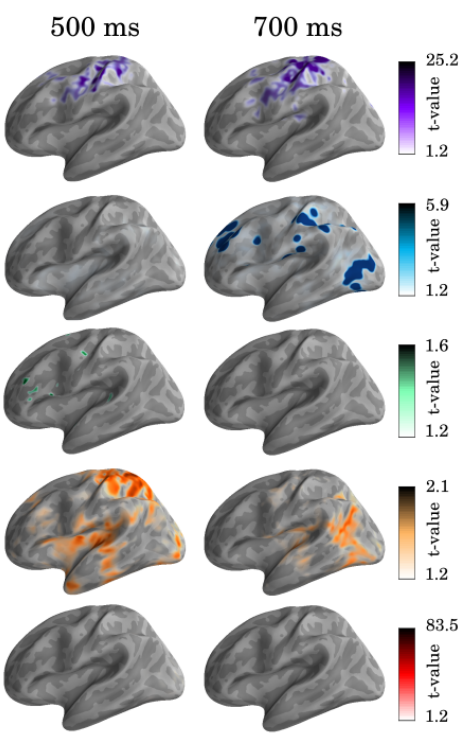

B

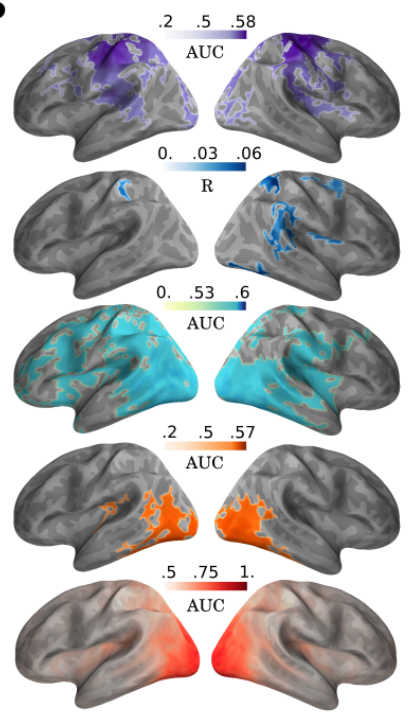

D

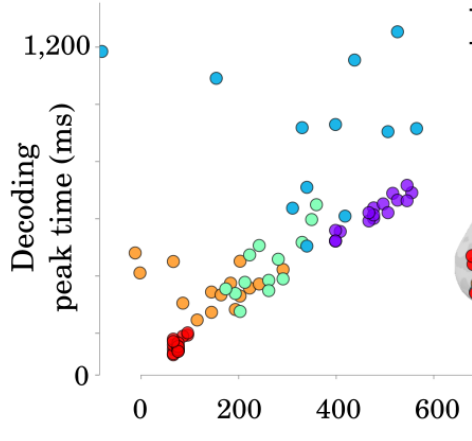

$\mathrm{E}$

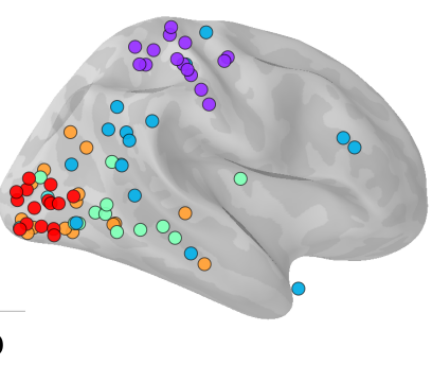

Figure 2: Spatio-temporal hierarchy. (A) Mass-univariate statistics. Each row plots the average-across-subjects beta coefficients obtained from regression between single-trial evoked activity and each of the five features orthogonally varying in this study. These results are displayed in Supplementary Video 2. Colors are thresholded based on t-values that exceed an uncorrected p <.1. (B) Spatial-decoders, consisting of linear models fit across all time sample for each source separately, summarize where each feature can be decoded. Lines indicate significant clusters of decoding scores across subjects. (C) Temporal-decoders, consisting of linear models fit across all MEG channels, for each time sample separately, summarize when each feature can be decoded. To highlight the sequential generation of each representation, decoding scores are normalized by their respective peaks. Additional statistics are available in Supplementary Figure 1. (D) The peak and the start of temporal decoding plotted for each subject (dot) and for each feature (color). (E) The peak spatial decoding plotted for each subject (dot) and for each feature (color).

tex but more anteriorly than stimulus identity $(\mathrm{x}=30.89 ; 175$ $y=-35.64 ; z=21.41 ; p<0.01)$. These mass-univariate ef- 176 fects did not survive correction for multiple compar- 177 isons (e.g. $210-320 \mathrm{~ms}: \bar{t}=1.79, \mathrm{p}=0.21$ ). Nonetheless, 178 spatial decoders, which mitigate the trade-off between 179 temporal specificity and the necessity to correct statis- 180 tical estimates for multiple comparisons, showed that ${ }_{181}$ perceptual category was reliably decoded from a large ${ }_{182}$ set of brain areas $(\bar{t}=4.82 ; \mathrm{p}<0.001 ; 594$ significant ver- 183 tices) (Fig.2G).

Importantly, when training the classifier on all ac- 185 tive trials to distinguish letters $(\mathrm{E} / \mathrm{H})$ and digits $(4 / 6),{ }_{186}$ we could significantly ( $\max \mathrm{AUC}=0.55 ; \mathrm{SEM}=0.011$; $\mathrm{p}<.01)$ decode this contrast for different unambiguous tokens $(\mathrm{A} / \mathrm{C}$ versus $9 / 8)$; suggesting that the response is tracking the abstract letter/digit contrast, abstracted from the specific pixel arrangement.

Fourth, trial difficulty (i.e. the distance between the presented stimulus and the closest unambiguous character) could be decoded between 270 and $1485 \mathrm{~ms}$ and peaked at $590 \mathrm{~ms}$ (12-regularized regression fit across sensors, $\mathrm{R}=0.12$; $\mathrm{SEM}=0.024 ; \mathrm{p}<0.01$ ). Difficulty signals were localized more anteriorly than those of stimulus category $(\mathrm{x}=12.58 ; \mathrm{y}=-91.44 ; \mathrm{z}=-1.23 ; \mathrm{p}<0.01)$. 
While spatial decoding led to significant clusters in ${ }_{237}$ the temporal, parietal and prefrontal areas (Fig.2B), the ${ }_{238}$ peak location of stimulus difficulty was highly variable ${ }_{239}$ across subjects and included the dorso-parietal cortex, 240 the temporo-parietal junction and the anterior cingulate ${ }_{241}$ cortex (Fig.2E).

Finally, temporal decoders of subjects' button press ${ }_{243}$ (left versus right index fingers) were significant from 244 $458 \mathrm{~ms}$ after stimulus onset and peaked at $604 \mathrm{~ms} 245$ (AUC $=0.85 ; \mathrm{SEM}=0.011 ; \mathrm{p}<0.001)$. A significant clus- 246 ter of motor signals could be detected around sen- 247 sorimotor cortices between 590 and $840 \mathrm{~ms}(\bar{t}=4.98$, 248 $\mathrm{p}<0.001$, Fig.2A). Response-lock analyses revealed 249 qualitatively similar but stronger results. For example, 250 temporal decoders were significant from $350 \mathrm{~ms}$ prior to 251 the response and up to $500 \mathrm{~ms}$ after the response reach- 252 ing an AUC of $94 \%$ at response time $(\mathrm{p}<0.001)$.

Overall, the time at which representations became 254 maximally decodable correlated with their peak loca- 255 tion along the postero-anterior axis (Fig.2D-E) $(\mathrm{r}=0.57,256$ $\mathrm{p}<0.001)$. These results thus strengthen the classic no- 257 tion that perceptual processes are hierarchically orga- 258 nized across space, time and function. Importantly how- 259 ever, this cascade of representations spreads over more 260 than $600 \mathrm{~ms}$ and largely exceeds the time it takes the 261 feedforward response to ignite the ventral and dorsal 262 pathways (Fig.1G and Supplementary Video 1).

\subsection{A hierarchy of recurrent layers explains the spatio- temporal dynamics of neural representations}

To clarify how a cascade of representations can be ${ }_{268}$ generated over extended time periods, we propose to 269 distinguish feedforward and/or recurrent architectures 270 depending on (i) the spatial location, (ii) the timing and 271 (iii) the spatio-temporal dynamics of their representa- 272 tions (Fig.3). This is done by simulating different ar- ${ }_{273}$ chitectures, and assessing their similarity to the MEG ${ }_{274}$ data. In these models, we assume that each 'layer' gen- 275 erates new hierarchical features, in order to account for 276 the organization of spatial decoders (Fig.2E). Further- 277 more, we only discuss architectures which can code for 278 all representations simultaneously, in order to account 279 for the overlapping temporal decoding scores (Fig.2C). 280 Finally, we only model discrete activations (i.e. a rep- 281 resentation is either encoded or not) as any more sub- 282 tle variation can be trivially accounted for by signal-to- ${ }_{283}$ noise ratio considerations.

Each model predicts (1) 'source' decoding time 285 courses (i.e. what is decodable within each layer) and 286 (2) 'temporal generalization' (TG) maps. TG is used to 287 characterize the dynamics of neural representations and ${ }_{288}$ consists in assessing the extent to which a temporal decoder trained at a given time sample generalizes to other time samples [24] (Fig.3D).

Our spatial and temporal decoding results can be accounted for by a feedforward architecture that both (i) generates new representations at each layer and (ii) propagates low-level representations across layers (Fig.3 Model 1: 'broadcast'). This architecture predicts that representations would not be maintained within brain areas. This lack of maintenance is not supported by our data. First, the position of the stimulus was decodable in the early visual cortex between $80-320 \mathrm{~ms}(\bar{t}=5.18, \mathrm{p}<.001)$ and thus longer than the stimulus presentation. Second, most temporal decoders significantly generalized over several hundreds of milliseconds (Fig.4A-B). For example, the temporal decoder trained to predict stimulus position from $\mathrm{t}=100 \mathrm{~ms}$ could accurately generalize until $\approx 500 \mathrm{~ms}$ as assessed with spatio-temporal cluster tests across subjects (Fig.4A). Similarly, temporal decoders of perceptual category and button-press generalized, on average, for $287 \mathrm{~ms}(\mathrm{SEM}=12.47 ; \mathrm{p}<.001)$ and $689 \mathrm{~ms}$ $(\mathrm{SEM}=30.94 ; \mathrm{p}<.001)$ respectively. Given that the neural activity underlying the decoded representations is partially stable over several hundreds of milliseconds, recurrent connections seem necessary to account our data (Fig.4 Model 2-4).

Consequently, we then considered a simple hierarchy of recurrent layers, where recurrence only maintains activated units (Fig.3 Model 2: 'maintain'). This architecture predicts strictly square TG matrices (i.e. temporal decoders would be equivalent to one another in terms of their performance) and is thus at odds with the largely diagonal TG matrices observed empirically (Fig.4A). Specifically, the duration of significant temporal decoding (fitting a new decoder at each time sample) was significantly longer than the generalization of a single decoder to subsequent time samples (e.g. 1,239 versus $287 \mathrm{~ms}$ for perceptual category $(\mathrm{t}=-61.39 ; \mathrm{p}<.001)$ and 1,215 versus $689 \mathrm{~ms}$ for button-press $(\mathrm{t}=-16.26$; $\mathrm{p}<.001)$, Fig.4B). These results thus suggest that the decoded representations depend on dynamically-changing activity: i.e. each feature is linearly coded by partially distinct brain activity patterns at different time samples.

It is difficult to determine, with MEG alone, whether such dynamic maintenance results from a change of neural activity within or across brain areas. Indeed, Model 1 and Model 3 can equally predict diagonal TG (Fig.3). However, these two models, and their combination (Model 4) diverge in terms of where information should be decodable. Specifically, source analyses revealed that both stimulus position and percep- 
A

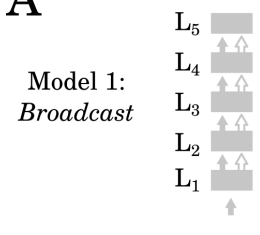

Model 2:
Maintain

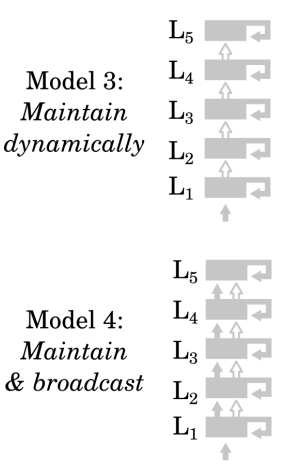

$\mathrm{L}_{5}$

$\mathrm{L}_{4}$

$\mathrm{L}_{3}$

$\mathrm{L}_{2}+\hat{ि}$

$\mathrm{L}_{1}$

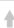

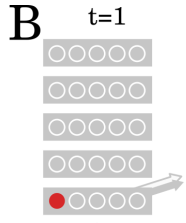

$t=2$
00000
00000
00000
00000

$\mathrm{t}=3$
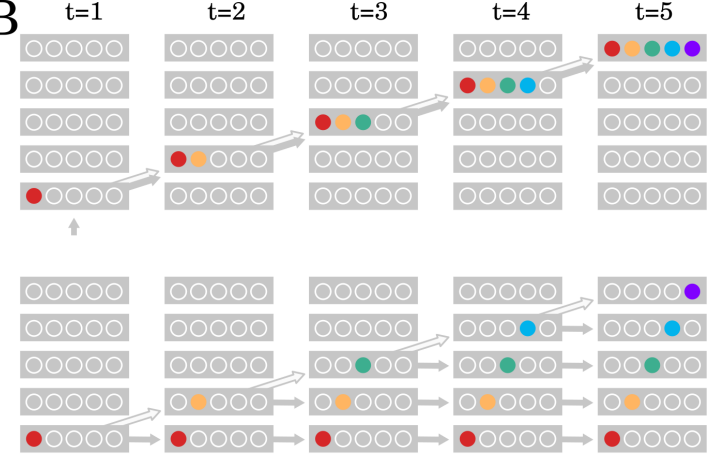

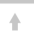

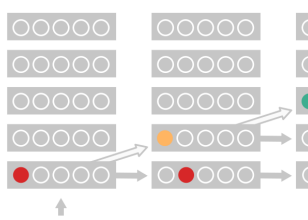

$\uparrow$
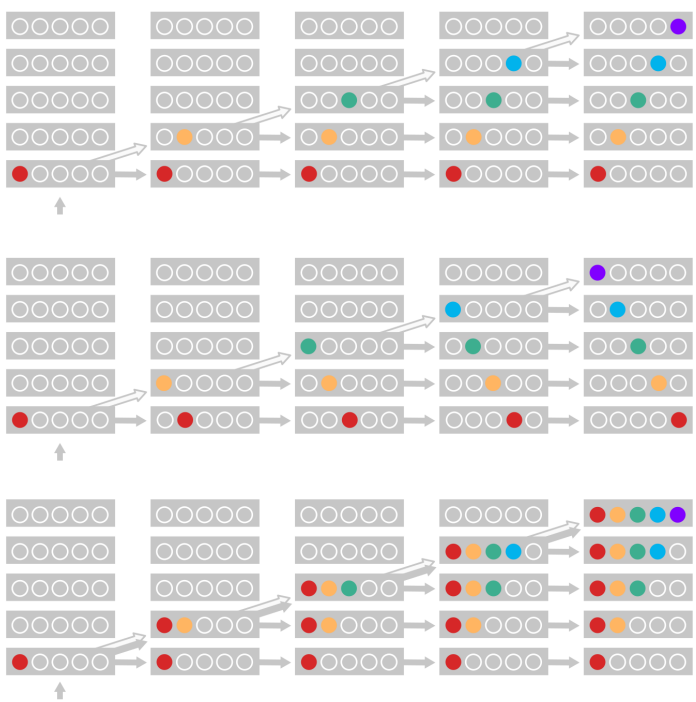

$00000 \rightarrow 0000 \rightarrow 0000$

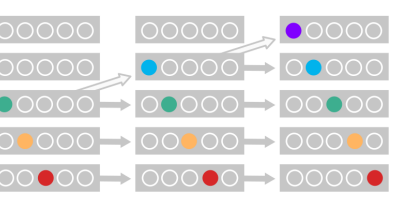

C
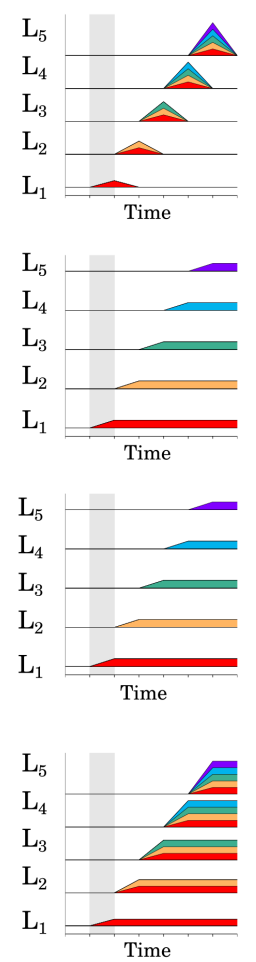

$\mathrm{D}$
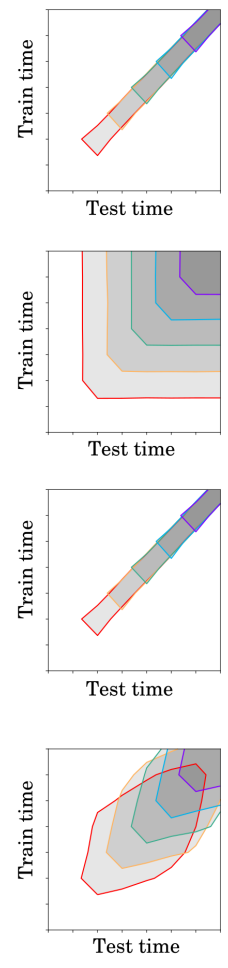

\begin{tabular}{|l|cc|}
\hline Copy feature & Layer & Position Identity Category \\
Make new feature & $\bigcirc$ Neuronal assembly & Difficulty \\
Button
\end{tabular}

Figure 3: Source and temporal generalization predictions for various neural architectures. (A) Four increasingly complex neural architectures compatible with the spatial and temporal decoders of Fig.2. For each model (rows), the five layers (L1, L2 ... L5) generates new representations. The models differ in their ability to (i) propagate low-level representations across the hierarchy, (ii) maintain information with each layer in a stable or dynamic way. (B) Activations within each layer plotted at five distinct time samples. Dot slots indicate different neural assemblies within the same layer. Colors indicate which feature is linearly represented. For clarity purposes, only effective connections are plotted between different time samples. (C) Summary of the information represented within each layer across time. (D) Expected result for of the temporal generalization analyses, based on the processing dynamics of each model.

tual category can be decoded across a wide variety of ${ }_{305}$ partially-overlapping brain areas (Fig.2B, Supplemen- 306 tary video 2), similarly to Model 4 . Nonetheless, our 307 MEG study remains limited in assessing whether within 308 brain regions dynamics also contribute to the diagonal 309 TG, which would suggest a mixture between models 3 310 and 4.

Together, source and TG analyses thus suggest that ${ }_{312}$ the slow and sequential generation of increasingly ab- ${ }_{313}$ stract representations depends on a hierarchy of recur- ${ }_{314}$ rent layers that generate, maintain and broadcast repre- ${ }_{315}$ sentations across the cortex.

\subsection{Hierarchical recurrence induces an accumulation of delays}

Can a hierarchy of recurrent processes account for 320 single-trial dynamics? To address this issue, we hy- 321 pothesized that recurrent processes would take variable amounts of time to converge to each intermediary representation. In this view, (i) each feature is predicted to propagate across brain areas at distinct moments, and (ii) the successive rise of decodable representations is thus predicted to incrementally correlate with reaction times (Fig.5A-E).

To test this hypothesis, we estimated how the peak of each temporal decoder varied with reaction times. For clarity purposes, we split reaction times into four quantiles, and averaged the time courses of temporal decoders relative to their training time. These analyses showed that the latencies of (i) perceptual category ( $\mathrm{r}=0.35 ; \mathrm{p}=0.006)$, (ii) stimulus difficulty $(\mathrm{r}=0.37$; $\mathrm{p}=0.004)$ and (iii) button press $(\mathrm{r}=0.66 ; \mathrm{p}<0.001)$ increasingly correlated with reaction times (Fig.5F-G).

Overall, these results show that we can track with 

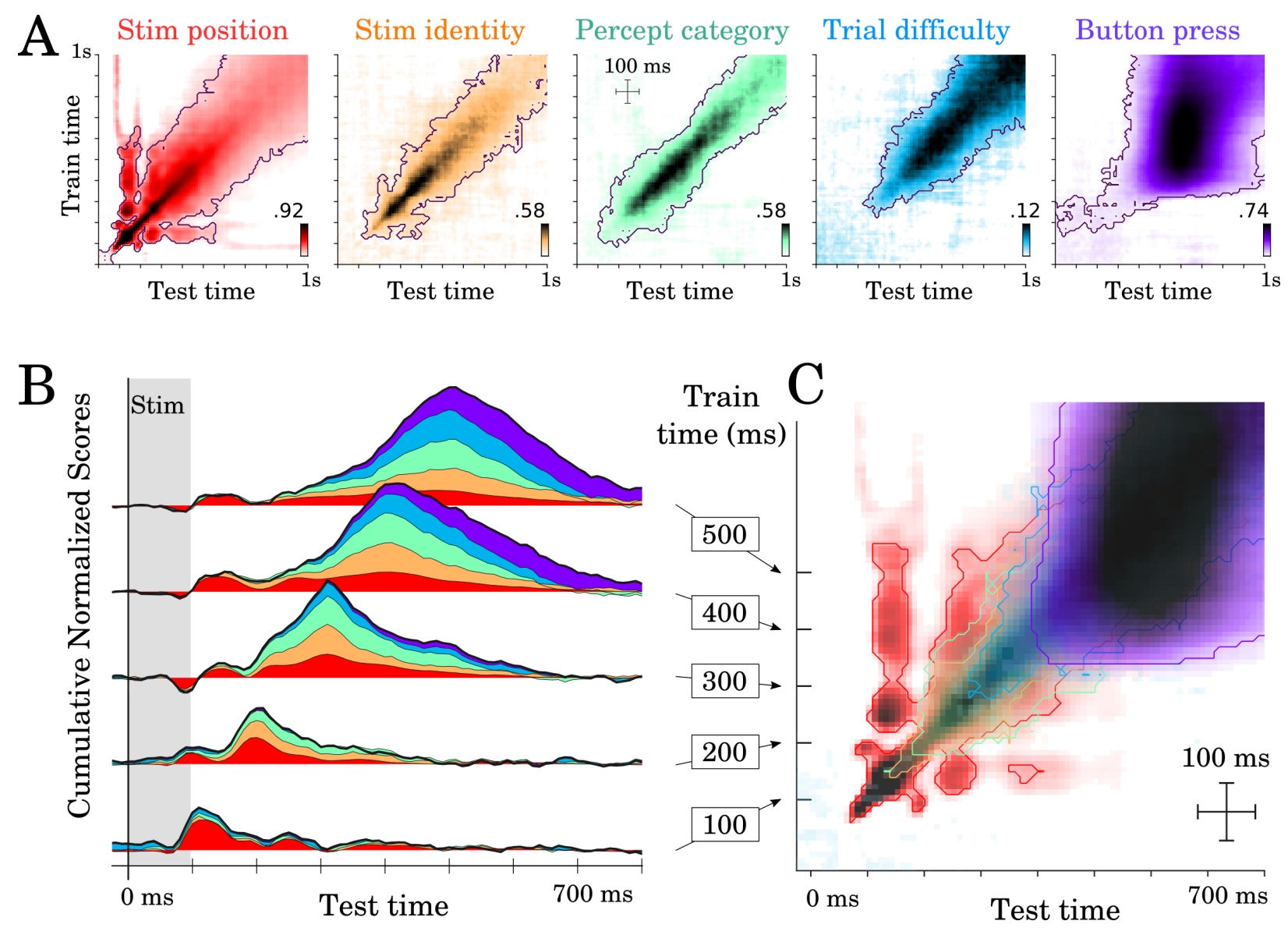

Figure 4: Temporal generalization results. (A) Temporal generalization for each of the five features orthogonally varying in our study. Color indicate decoding score (white=chance). Contours indicate significant decoding clusters across subjects. (B) Cumulative temporal generalization scores for the temporal decoders trained at 100, 200, 300, 400 and 500 ms respectively. These decoding scores are normalized by mean decoding peak for clarity purposes. (C) Same data as A but overlaid. For clarity purposes, contours highlight the 25th percentile of decoding performance.

MEG, a series of decisions generated by hierarchical recurrent processes. This neural architecture partially 339 accounts for subjects' variable and relatively-slow reac- 340 tion times.

\subsection{Hierarchical recurrence implements a series of all- or-none decisions}

An architecture based on successive decisions pre- 346 dicts a loss of ambiguous information akin to all-or- ${ }_{347}$ none categorization across successive processing stages 348 (Fig.6A). To test this prediction, we quantified the ex- 349 tent to which the decoding of 'percept category' and ${ }_{350}$ of 'motor action' varied linearly or categorically with ${ }_{351}$ (i) categorical evidence and (ii) motor evidence respec- 352 tively (i.e. the extent to which the stimulus (i) objec- ${ }_{353}$ tively looks like a letter or a digit and (ii) should have ${ }_{354}$ led to a left or right button press given its pixels).
The probabilistic decoding predictions of percept category correlated linearly with sensory evidence be-

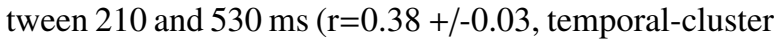
$\mathrm{p}<0.001)$. The spatial decoders fit from 200 to $400 \mathrm{~ms}$ clustered around the VWFA ( $\bar{t}=4.6 ; \mathrm{p}=.02 ; 224$ vertices) (Fig 6H). These results suggest that this region first represents the stimulus objectively (i.e. in its full ambiguity).

Between 400 and $810 \mathrm{~ms}$, the predictions of 'perceptual category' decoders were better accounted for by sigmoidal $(\mathrm{r}=0.77+/-0.03,199 \mathrm{p}<0.001)$ than by linear trends $(\mathrm{r}=0.77+/-0.03, \mathrm{p}<0.001)$. Spatial decoding analyses restricted to the $500-700 \mathrm{~ms}$ time window was more distributed $(\bar{t}=4.4 ; \mathrm{p}=.022 ; 110$ vertices $)$. Finally, ambiguous stimuli (steps 5 and 6 on the continuum) reached maximum decodability 205 ms later than unambiguous stimuli (steps 1 and 8) $(\mathrm{p}<0.001)$ (Fig.6J). The interaction between trend (linear or sigmoidal) and 


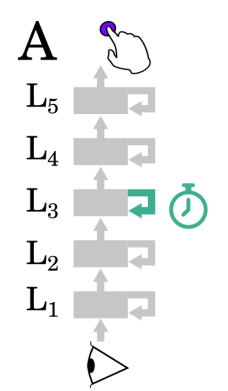

B

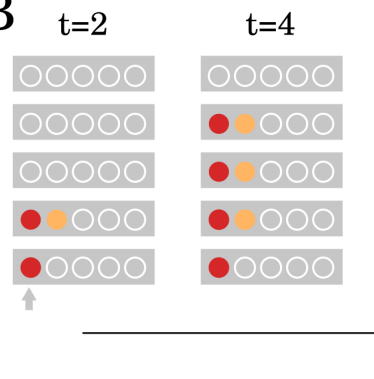

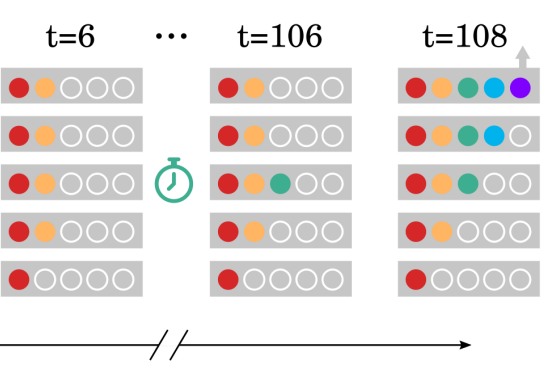

Motor delay

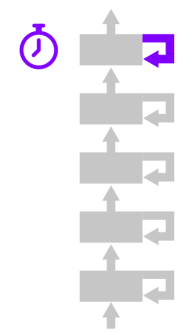

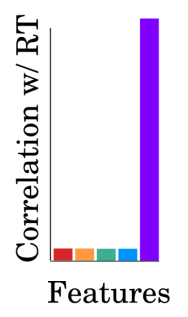

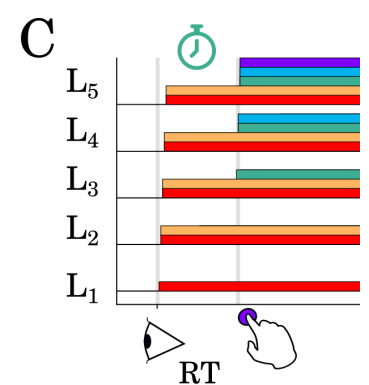

Cumulative delay

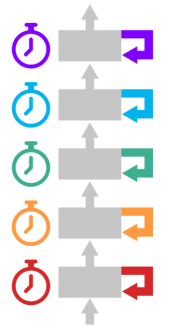

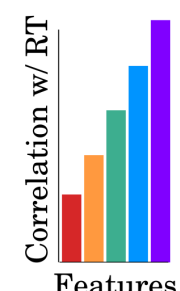

Features
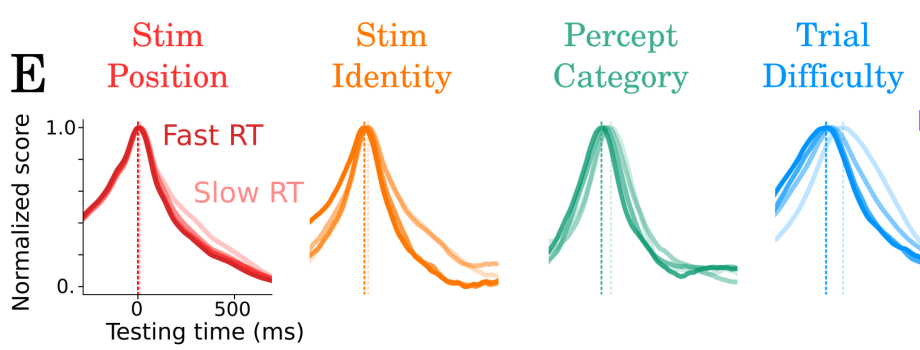

\section{Button \\ Press}

Fast RT

\section{G}

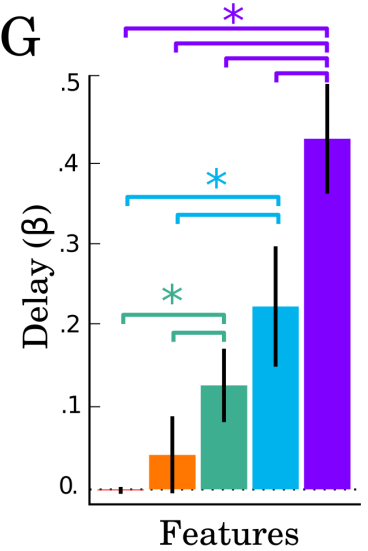

Figure 5: Correlation between TG peaks and reaction times. (A, B) Recurrent processing at a given processing stage is hypothesized to take a variable amount of time to generate adequate representations. (C) According to this hypothesis, the rise of the corresponding and subsequent representations would correlate with reaction times. (D, left) Predictions when delays are only induced by the perceptual stage of processing. (D, middle) Predictions when delays are only induced by the motor processing processing stage. (D, right) Predictions when delays are induced by all processing stages. (E) TG scores aligned to training time, split into trials within the fastest and slowest reaction-time quantile and averaged across reaction times bins. Dark and light lines indicate the average decoding performance for trials with fastest and slowest reaction times respectively. (F) Each subject (dot) mean peak decoding time (y-axis) as a function of reaction time (x-axis) color-coded from dark (fastest) to light (slowest). The beta coefficients indicate the average delay estimate. (G) The average slope between processing delay and reaction time for each feature. Error-bars indicate the SEM.

window latency was significant across subjects $(\mathrm{r}=0.07 ; 364$ $\mathrm{SEM}=0.01 ; \mathrm{p}=0.002$ ).

This progressive categorization of the letter/digit rep- 366 resentations contrasts with the all-or-none pattern of motor signals. Specifically, the probabilistic predictions ${ }_{367}$ of button-press decoders varied categorically with re- 368 sponse evidence from 440 to $1,290 \mathrm{~ms}$ (sigmoid $>$ linear 369 cluster, $\bar{t}=3.17 ; \mathrm{p}<0.001)$. There was also a more tran- 370 sient linear trend from 410 to $580 \mathrm{~ms}(\bar{t}=3.69 ; \mathrm{p}<0.001)$. This suggests that, unlike perceptual category, motor signals largely derive from categorical inputs.

Together, delay (Fig.5) and categorization (Fig. 6) analyses thus show that perceptual representations slowly become categorical and are subsequently followed by all-or-none motor representations. 
A

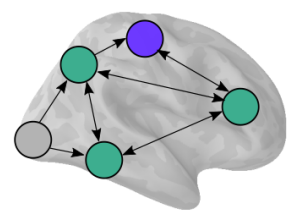

Sensory categorization

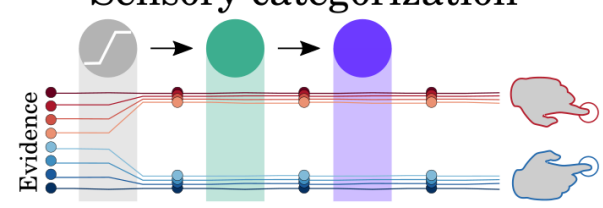

Perceptual categorization

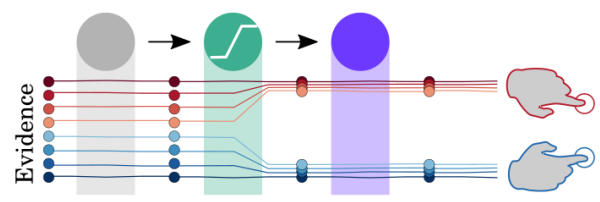

Motor categorization

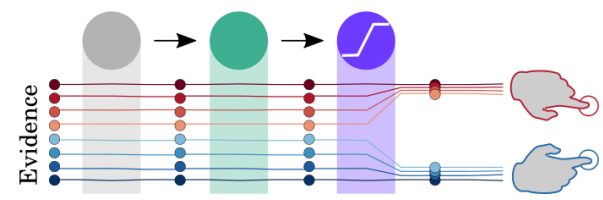

B

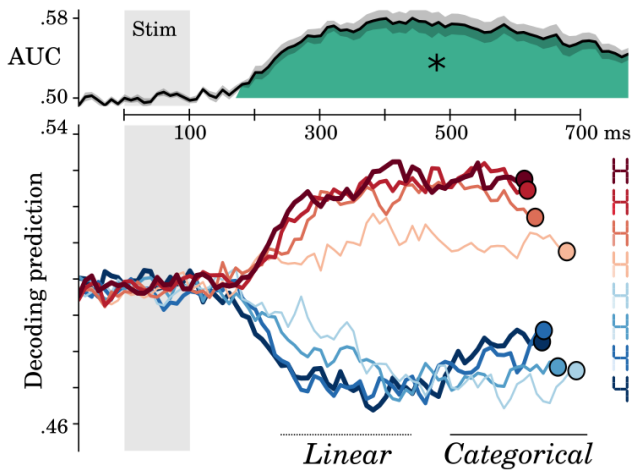

$\mathrm{D}$

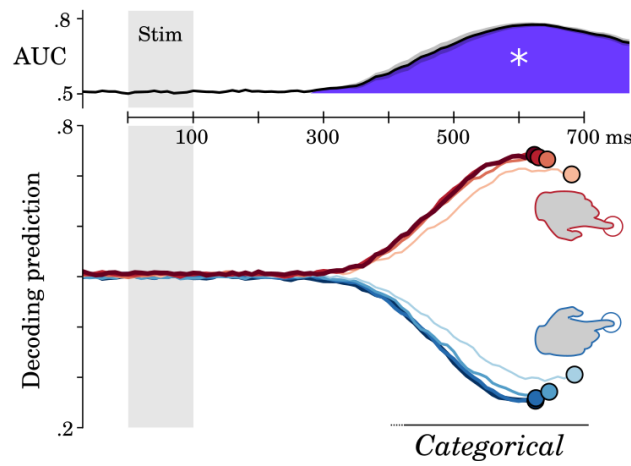

C

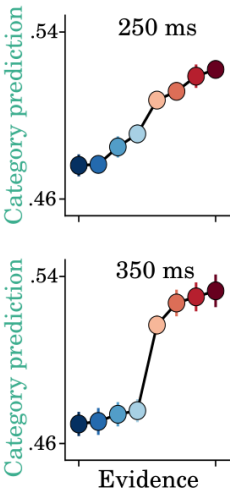

$\mathrm{E}$

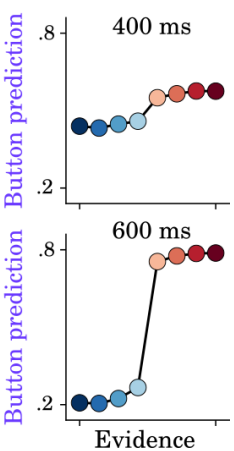

Figure 6: Motor and perceptual decisions. (A) Hypothesis space for when responses become categorical: during sensory, perceptual or motor processing. (B, top) Time course of decoding the perceptual decision. (B, bottom) Classifier predictions split into different levels of sensory evidence. (C) Averaging probabilities in different time-windows shows the linear-categorical shift in how information is represented. (D, top) Time course of decoding the motor decision. (D, bottom) Splitting classifier predictions into different levels of difficulty. (E) Different windows of classifier predictions, showing the categorical responses throughout processing.

\section{Discussion}

Our results show that briefly-flashed stimuli elicit a 391 cascade of representations that spread well beyond the 392 initial feedforward recruitment of the visual pathways. 393 A hierarchy of recurrent processes that incrementally 394 build representations best explains this cascade, its ac- 395 cumulated delays and its all-or-none categorizations. 396

While macroscopic MEG signals advantageously 397 give a birds-eye view of the cortical correlates of per- 398 ceptual decision making, it should be stressed that their 399 source reconstruction remains a coarse approximation. 400 Consequently, identifying (1) the role of subcortical ar- 401 eas and (2) the extent to which representations dynami- ${ }_{402}$ cally change within each brain area will necessitate in- 403 vasive brain recordings.

Nonetheless, our results bridge three important lines 405 of research on the neural and computational bases of 406 visual processing.
First, core-object recognition research, generally based on $\approx 100$ ms-long image presentations has repeatedly shown that the spiking responses of the inferotemporal cortex is better explained by recurrent models than by feedforward ones [9, 14]. In particular, Kar et al have recently shown that images that are challenging to recognize, lead to delayed content-specific spiking activity in the macaque's infero-temporal cortex [14]. Our findings, based on simpler but highly-controlled stimuli, are consistent with these results and further highlight that perceptual representations are not confined to the inferotemporal cortices, but also reach a large variety of parietal and prefrontal areas [25].

Second, the present study makes important contributions to the perceptual decision making literature $[11,13]$. With some notable exceptions (e.g. [26]), this line of research primarily aims to isolate motor and supra-modal decision signals in the presence of sustained visual inputs: i.e. neural responses ramping to- 
wards a virtual decision threshold, independently of the ${ }^{454}$ representation on which this decision is based [13]. The ${ }_{455}$ present study complements this approach by tracking 456 the representation-specific signals that slowly emerge ${ }^{457}$ after a brief stimulus. Our results thus open an ex- 458 citing avenue for querying the gating mechanisms of 459 successive decisions and clarifying the role of the pre- 460 frontal areas in the coordination multiple perceptual and ${ }_{461}$ supramodal modules [27].

Finally, our results constitute an important confirma- 463 tion of modern theories of perception. In particular, the ${ }^{464}$ Global Neuronal Workspace Theory predicts that per- 465 ceptual representations need to be broadcast to asso- 466 ciative cortices via the fronto-parietal areas to lead to 467 subjective reports [8]. Yet, at some notable exceptions 468 $[28,29]$, previous studies often fail to dissociate percep- 469 tual contents and perceptual reports (e.g. [30, 31]). By 470 contrast, the present experimental design allows an un- 471 precedented dissection of the distinct processing stages 472 that transform sensory input into perceptual representations and, ultimately, actions. The generation of let- 473 ter and digit representations in the dedicated brain areas 474 $[32,17]$ and their subsequent broadcast to the cortex re- ${ }^{475}$ inforce the notion that subjective perception relate to the ${ }^{476}$ global sharing of content-specific representations across 477 brain areas $[8,33]$.

\section{Method}

\subsection{Target stimuli}

Using the font designed in [15], the stimuli were made from $0,4,5,6,8,9, \mathrm{~A}, \mathrm{C}, \mathrm{E}, \mathrm{H}, \mathrm{O}, \mathrm{S}$, or from a linear combination of two of these characters varying in a single black bar (hereafter 'pixel'). The corresponding 'morphs' were created by adjusting the contrast of the remaining pixel along eight equally spaced steps between 0 (no bar) and 1 (black bar).

\subsection{Experiment 1}

Eight subjects with normal or corrected vision, seated $\approx 60 \mathrm{~cm}$ from a 19 " CRT monitor $(60 \mathrm{~Hz}$ refresh rate, resolution: 1024x768), performed a stimulus identification task with continuous judgements across 28 variably ambiguous stimuli generated from digit stimuli. Ten euros were provided in compensation for this 1-hour experiment.

Subjects performed four blocks of 50 trials, each or- ${ }_{485}$ ganized in the following way. After a 200 ms fixation, a 486 target stimulus, randomly selected from one of the 28 stimuli, was flashed for $83 \mathrm{~ms}$ on a $50 \%$ gray background to the left or to the right of fixation. The orientation of the reporting disk (e.g 5-6-8-9 versus 5-9$8-6)$ was counterbalanced across subjects. Subjects had then up to 10 seconds to move a cursor on a large disk to report their percepts. The radius on the disk indicated subjective visibility (center=did not see the stimulus, disk border $=\max$ visibility). The angle on the disk indicated subjective identity (e.g. 5, 6, 8, 9 for the top left, top right, bottom right, and bottom left 'corners' respectively). Inter-trial interval was $500 \mathrm{~ms}$. To verify that subjects provided meaningful reports, the target stimulus was absent $15 \%$ of the trials. Absent trials were rated with a low visibility (i.e radius below $5 \%$ of the disk radius) in most cases. Absent trials and trials reported with a low visibility were excluded from subsequent analyses. The report distribution plotted in Fig.1B were generated with Seaborn's bivariate Gaussian kernel density estimate function with default parameters.

Modeling categorical reports. To test whether subjective reports of stimulus identity varied linearly or categorically with sensory evidence, we analyzed how reports' angle (i.e. subjective identity) varied with the expected angle given the stimulus (i.e. sensory evidence).

For each morph (5-6, 5-8, 9-8 and 6-8) separately, we fit a linear model:

$$
\hat{y} \leftarrow \beta_{1} x+\beta_{0}
$$

and a sigmoidal model:

$$
\hat{y} \leftarrow \frac{1}{1+\exp \left(\beta_{1} x+\beta_{2}\right)}+\beta_{0}
$$

where $\hat{y}$ is the report angle predicted by the model, $x$ is expected angle given the stimulus pixels and $\beta_{0}$ is a free bias parameter.

To minimize the effects of noise, behavioral reports were first averaged within each level of evidence, sorted from the stimulus with the least pixels (e.g. 5, in 5-6 morph) to the stimulus with the most pixels (e.g. 6 in the 5-6 morph). The resulting averages were normalized to range between 0 and 1 within each subject. The $\beta$ parameters were fit with Scipy's 'curve_fit' function [34] to minimize a mean squared error across trials $i$ :

$$
\underset{\beta}{\operatorname{argmin}} \sum_{i}\left(y_{i}-\hat{y}_{i}\right)^{2}
$$

Because the linear and sigmoidal models have distinct numbers of free parameters, we compared them within a 5-split cross-validation. Specifically, the two models were repeatedly fit and tested on independent 
trials. A Pearson correlation coefficient $r$ summarised ${ }_{539}$ the ability of each model to accurately predict $y_{\text {test }}$ given ${ }_{540}$ $x_{\text {train }}, y_{\text {train }}$ and $x_{\text {test }}$. Finally, a Wilcoxon test was ap- ${ }_{541}$ plied across subjects to test whether the two models ${ }_{542}$ were consistently above chance $(r>0)$ and consistently ${ }_{543}$ different from one another $\left(r_{\text {sigmoid }}>r_{\text {linear }}\right)$.

Experiment 2. This experiment was performed at Neurospin, Gif usr Yvette, thanks to the support of Stanislas Dehaene. Seventeen subjects performed a discrete identification task across 22 variably ambiguous stimuli generated from letters and digits inside an Elekta Neuromag MEG scanner (204 planar gradiometers and 102 magnetometers). Seventy euros were provided in compensation to the 1-hour experiment and 30 minutes of preparation.

Participants' head shape was digitized along with five fiducial points on the forehead and on each aural canal. ${ }^{553}$ Five head-position coils were placed on subjects head ${ }^{554}$ and localized at the beginning of each block.

The trial structure was as follows. A black fixation ${ }^{556}$ cross was displayed on a 50\% gray background for $300{ }^{557}$ ms followed by a $100 \mathrm{~ms}$-long target stimulus presented ${ }^{558}$ on the left or on the right of fixation. Two task-irrelevant ${ }^{559}$ flankers (e.g. stimulus can be read as an S or a 5) were ${ }^{560}$ displayed on the side of this target stimulus to increase ${ }^{561}$ our chances of eliciting recurrent processing via crowd- 562 ing [18]. Subjects were given two seconds to report the ${ }^{563}$ identity of the stimulus. Reports of stimulus identity ${ }^{564}$ were given by pressing a button with the left and right ${ }^{565}$ index fingers respectively. The identity-button mapping ${ }^{56}$ changed on every block to orthogonalize the neural cor- ${ }^{567}$ relates of stimulus identity and the neural correlates of ${ }^{568}$ motor actions. For example, in block 1, perceiving an ${ }^{569}$ E or a 4 should have been reported with a left button ${ }^{570}$ press, whereas in block 2, E and 4 should have been ${ }^{57}$ reported with a right button press. The identity-button was explicitly reminded before each block. In addition, ${ }^{572}$ a visual feedback was displayed after non-ambiguous 573 trials. Specifically, the fixation turned green for $100 \mathrm{~ms} 574$ or red for $300 \mathrm{~ms}$ in correct and incorrect trials respec- 575 tively. The brain responses to these feedback stimula- 576 tions are not analyzed in the present study. Inter-trial 577 interval was 1 second. Subjects were provided a short 578 training to ensure they understood the task, and identi- 579 fied non-ambiguous targets at least $80 \%$ of the time. $\quad 580$

A total of 1920 trials, grouped into 40 blocks, were ${ }_{581}$ performed by each subject, 320 of which were presented 582 passively at the end of each block - subjects were not ${ }_{583}$ required to provide a response. The trial structure was 584 generated by (i) permuting all combinations of stimulus 585 features (e.g. position, identity, response mapping, dif- 586 ficulty), and (ii) shuffling the order of presentation for each subject. The experiment was presented using Psychtoolbox [35].

All experiments were approved by the local ethics committee. All subjects signed an informed consent form.

\subsection{Structural MRI}

For each subject, an anatomical MRI with a resolution of $1 \times 1 \times 1.1 \mathrm{~mm}$ was acquired after the MEG experiment with a 3T Siemens scanner. Gray and white matter were segmented with Freesurfer 'recon-all' pipeline [20] and coregistered with each subject's digitized head shapes along with fiducial points.

\subsection{Preprocessing}

The continuous MEG recording was noise-reduced using Maxfilter's SSS correction on the raw data, bandpass-filtered between 0.5 and $40 \mathrm{~Hz}$ using MNEPython's default parameters with firwin design [21] and downsampled to $250 \mathrm{~Hz}$. Epochs were then segmented between $-300 \mathrm{~ms}$ and $+1500 \mathrm{~ms}$ relative to stimulus onsets.

After coregistering the MEG sensor data with subjects' structural MRI and the head position coils, we computed the forward model using a 1-layer (inner skull) boundary element model, for each subject separately and fit a minimum-norm inverse model (signal to noise ratio: 3 , loose dipole fitting: 0.2 , with normal orientation of the dipole relative to the cortical sheet) using the noise covariance across sensors averaged over the pre-stimulus baseline across trials. Finally, the inverse model was applied to single-trial data resulting in a dynamic Statistical Parameter Map (dSPM) [19] value for each source at each time sample.

\subsection{Modeled features}

We investigated whether single-trial source and sensor evoked responses varied as a function of five features: (1) the position of the stimulus on the computer screen (left versus right of fixation), (2) the morph from which the stimulus is generated (E-6 versus H-4), (3) the category of the stimulus (letter versus digit), (4) the difficulty of the trial (maximum difficulty = stimuli with pixel at 50\% contrast; minimum difficult stimuli with pixels at $0 \%$ or $100 \%$ contrast), and (5) the response button used to report the stimulus (left versus right button). By design, these five features are independent of one another.

It is challenging to dissociate brain responses that represent objective sensory information from those that 
represent perceptual decisions as the two are generally 615 collinear. To address this issue, we first fit univariate and ${ }_{616}$ multivariate models to predict perceptual category: i.e. 617 whether the button press indicated a character that be- ${ }_{618}$ longs to the digit or to the letter category. This feature is 619 independent of the button press (e.g. the letter $\mathrm{E}$ and the ${ }_{620}$ digit 4 can be reported with the same button in a given ${ }_{621}$ block). Furthermore, this feature is not necessary to per- 622 form the task (i.e. knowing whether $\mathrm{E}$ and $\mathrm{H}$ are letters ${ }_{623}$ is unnecessary to discriminate them). We reasoned that ${ }_{624}$ if subjects automatically generates letter/digit represen- 625 tations during perceptual categorization, then we should ${ }_{626}$ be able to track the generation of this abstract feature ${ }_{627}$ from brain activity.

\subsection{Mass univariate statistics}

To estimate whether brain responses correlated with ${ }^{631}$ each of these five features, we first fit, within each sub- 632 ject, mass univariate analyses at each source location ${ }^{633}$ and for each time sample with a linear regression:

$$
\beta=\left(X^{T} X\right)^{-1} X y
$$

where $X \in \mathbb{R}^{n, f}$ is a design matrix of $n$ epochs by ${ }^{637}$ $f=5$ features and $y \in \mathbb{R}^{n}$ is the univariate brain re- ${ }_{639}$ sponse at a given source and at given time. The effect 640 sizes $\beta$ were then passed to second-level statistics across ${ }_{641}$ subjects corrected for multiple comparisons using non- ${ }_{642}$ parametric spatio-temporal cluster testing (see below).

\subsection{Decoding}

Decoding analyses consists in predicting each feature from multivariate brain responses. Decoding analyses were performed within a 5-split stratified K-Fold crossvalidation using 12-regularized linear models. Classifiers consisted of logistic regressions (with scikit-learn [36]'s default parameters: $C=1$ ):

$$
\underset{\beta}{\operatorname{argmin}} \sum_{i} \log \left(1+\exp \left(-y_{i} \beta^{T} \vec{x}_{i}\right)\right)+C\|\beta\|^{2}
$$

where $y_{i} \in\{ \pm 1\}$ is the feature to be decoded at trial $i$ and $x_{i}$ is the multivariate brain response.

Regressors consisted of ridge regression (with scikitlearn [36]'s default parameters: $\alpha=1$ ).

$$
\underset{\beta}{\operatorname{argmin}} \sum_{i}\left(y_{i} \beta^{T} x_{i}\right)^{2}+\alpha\|\beta\|^{2}
$$

For each subject independently, decoding perfor- 65 mance was summarized across trials, with an area under 653 the curve (AUC) and a Spearman $r$ correlation score for 654 classifiers and regressors respectively.
All decoders were provided with data normalized by the mean and the standard deviation in the training set.

Spatial decoding consists in fitting a series of decoders at each brain source independently, across all 1,500 time samples relative to stimulus onset. This analysis results in a decoding brain map that indicates where a feature can be linearly decoded in the brain. These decoding maps were then passed to cluster-corrected second-level statistics across subjects.

Temporal decoding consists in fitting a series of decoders at each time sample independently, across all 306 MEG sensors. This analysis results in a decoding time course that indicates when a feature can be linearly decoded from MEG signals. These decoding time courses were then passed to cluster-corrected secondlevel statistics across subjects.

Temporal generalization (TG) consists in testing whether a temporal decoder fit on a training set at time $t$ can decode a testing set at time $t^{\prime}$ [24]. TG can be summarized with a square training time $\times$ testing time decoding matrix. To quantify the stability of neural representations, we measured the duration of above-chance generalization of each temporal decoder. To quantify the dynamics of neural representations, we compared the mean duration of above-chance generalization across temporal decoders to the duration of abovechance temporal decoding (i.e. the diagonal of the matrix versus its rows). These two metrics were assessed within each subject and tested with second-level statistics across subjects.

\subsection{Linear versus Categorical}

To test whether neural representations varied as a function of (i) reaction times (RTs, split into 4 quantiles), (ii) sensory evidence (i.e. the extent to the stimulus objectively corresponds to a letter) and (iii) motor evidence (i.e. whether the stimulus should have led to the left button press), we analyzed the extent to which decoders' predictions covaried with each of these three variables $z$ :

$$
f\left(z, \beta^{T} X\right)
$$

where $f$ is a linear or a sigmoidal model, $X$ is the multivariate brain response and $\beta$ is the decoder's coefficient fit with cross-validation.

\subsection{Statistics}

Univariate, decoding and TG models were fit within subjects, and tested across subjects. In case of repeated estimates (e.g. temporal decoding is repeated at each time sample), statistics derived from non-parametric cluster-testing with 10,000 permutations across subjects with MNE-Python's default parameters [21]. 
Simulations. To formalize how distinct neural architectures lead to distinct spatio-temporal dynamics, we modeled discrete linear dynamical systems forced with a transient input $U$. Specifically:

$$
X_{t+1}=A X_{t}+B U_{t}
$$

where $\mathrm{X}$ is a multidimensional times series (i.e. neurons $\mathrm{x}$ time), $\mathrm{A}$ is the architecture, and corresponds to square connectivity matrix (i.e. neurons $\mathrm{x}$ neurons), B is an input connectivity matrix (i.e. inputs x neurons), and $\mathrm{U}$ is the input vector.

Distinct architectures differ in the way units are connected with one another. For simplicity purposes, we order units in the $A$ matrix such that their row index correspond to their hierarchical levels.

In this view, the recurrent, feedforward and skip connections of the architecture A were modeled as a binary diagonal matrix $R$, a shift matrix $F$ and a matrix $S$ with 1 entries in the last column respectively. These three matrices were modulated by specific weights, as detailed below. The input $U$ was only connected to the first "processing stage", i.e. to the first unit(s) of $A$, via a matrix $B$ constant across architectures, and consisted of a transient square-wave input, that mimics the transient flash of the stimulus onto subjects' retina.

To model multiple features, we adopted the same procedure with multiple units per layer. Each unit within each layer was forced to encode a specific feature.

Each architecture was fed an input at $\mathrm{t}=1$, and simulated for 8 time steps. Finally, temporal generalization analyses based on the architectures' activations were applied for each of the features.

\section{Acknowledgement}

This project received funding from the European Union's Horizon 2020 research and innovation program under grant agreement No 660086, the BettencourtSchueller Foundation, the Fondation Roger de Spoelberch, the Philippe Foundation and the Abu Dhabi Institute G1001. We are infinitely grateful to Stanislas Dehaene for his support. 
bioRxiv preprint doi: https://doi.org/10.1101/840074; this version posted November 12, 2019. The copyright holder for this preprint (which was not certified by peer review) is the author/funder, who has granted bioRxiv a license to display the preprint in perpetuity. It is made available under aCC-BY-NC-ND 4.0 International license.

[1] Hubel, D. H. \& Wiesel, T. N. Receptive fields, binocular inter- 759 action and functional architecture in the cat's visual cortex. The 760 Journal of physiology 160, 106-154 (1962).

[2] Maunsell, J. \& van Essen, D. C. The connections of the mid- 762 dle temporal visual area $(\mathrm{mt})$ and their relationship to a cortical 763 hierarchy in the macaque monkey. Journal of Neuroscience 3, 764 2563-2586 (1983).

[3] Riesenhuber, M. \& Poggio, T. Hierarchical models of object 766 recognition in cortex. Nature neuroscience 2, 1019 (1999).

[4] DiCarlo, J. J., Zoccolan, D. \& Rust, N. C. How does the brain 768 solve visual object recognition? Neuron 73, 415-434 (2012). $\quad 769$

[5] Yamins, D. L. et al. Performance-optimized hierarchical models 770 predict neural responses in higher visual cortex. Proceedings of 771 the National Academy of Sciences 111, 8619-8624 (2014).

[6] Schrimpf, M. et al. Using brain-score to evaluate and build 773 neural networks for brain-like object recognition. In Cosyne 774 19, Date: 2019/02/28-2019/03/03, Location: Lisbon, Portugal 775 (2019).

[7] Ratcliff, R. \& Smith, P. L. A comparison of sequential sampling 777 models for two-choice reaction time. Psychological review 111, 778 333 (2004).

[8] Dehaene, S. \& Changeux, J.-P. Experimental and theoretical ap- 780 proaches to conscious processing. Neuron 70, 200-227 (2011). 781

[9] Lamme, V. A. \& Roelfsema, P. R. The distinct modes of vi- 782 sion offered by feedforward and recurrent processing. Trends in 783 neurosciences 23, 571-579 (2000).

[10] Gray, C. M., König, P., Engel, A. K. \& Singer, W. Oscillatory 785 responses in cat visual cortex exhibit inter-columnar synchro- 786 nization which reflects global stimulus properties. Nature 338, 787 334 (1989).

[11] Gold, J. I. \& Shadlen, M. N. The neural basis of decision mak- 789 ing. Annual review of neuroscience $\mathbf{3 0}$ (2007).

[12] Shadlen, M. N. \& Newsome, W. T. Neural basis of a perceptual 791 decision in the parietal cortex (area lip) of the rhesus monkey. 792 Journal of neurophysiology 86, 1916-1936 (2001).

[13] O'connell, R. G., Dockree, P. M. \& Kelly, S. P. A supramodal accumulation-to-bound signal that determines perceptual decisions in humans. Nature neuroscience 15, 1729 (2012).

[14] Kar, K., Kubilius, J., Schmidt, K., Issa, E. B. \& DiCarlo, J. J. Evidence that recurrent circuits are critical to the ventral stream's execution of core object recognition behavior. Nature neuroscience 22, 974 (2019).

[15] King, J.-R. \& Dehaene, S. A model of subjective report and objective discrimination as categorical decisions in a vast representational space. Philosophical Transactions of the Royal Society B: Biological Sciences 369, 20130204 (2014).

[16] Dehaene, S. \& Cohen, L. The unique role of the visual word form area in reading. Trends in cognitive sciences 15, 254-262 (2011).

[17] Shum, J. et al. A brain area for visual numerals. Journal of Neuroscience 33, 6709-6715 (2013).

[18] Strasburger, H., Rentschler, I. \& Jüttner, M. Peripheral vision and pattern recognition: A review. Journal of vision 11, 13-13 (2011).

[19] Dale, A. M. et al. Dynamic statistical parametric mapping: combining fmri and meg for high-resolution imaging of cortical activity. Neuron 26, 55-67 (2000).

[20] Fischl, B. Freesurfer. Neuroimage 62, 774-781 (2012).

[21] Gramfort, A. et al. Mne software for processing meg and eeg data. Neuroimage 86, 446-460 (2014).

[22] Hagler Jr, D. J. \& Sereno, M. I. Spatial maps in frontal and prefrontal cortex. Neuroimage 29, 567-577 (2006).

[23] Wandell, B. A., Dumoulin, S. O. \& Brewer, A. A. Visual field maps in human cortex. Neuron 56, 366-383 (2007).

[24] King, J. \& Dehaene, S. Characterizing the dynamics of mental representations: the temporal generalization method. Trends in cognitive sciences 18, 203-210 (2014).

[25] Freedman, D. J. \& Miller, E. K. Neural mechanisms of visual categorization: insights from neurophysiology. Neuroscience $\mathcal{E}$ Biobehavioral Reviews 32, 311-329 (2008).

[26] Philiastides, M. G. \& Sajda, P. Eeg-informed fmri reveals spatiotemporal characteristics of perceptual decision making. Journal of Neuroscience 27, 13082-13091 (2007).

[27] Sarafyazd, M. \& Jazayeri, M. Hierarchical reasoning by neural circuits in the frontal cortex. Science 364, eaav8911 (2019).

[28] Tong, F., Nakayama, K., Vaughan, J. T. \& Kanwisher, N. Binocular rivalry and visual awareness in human extrastriate cortex. Neuron 21, 753-759 (1998).

[29] King, J.-R., Pescetelli, N. \& Dehaene, S. Brain mechanisms underlying the brief maintenance of seen and unseen sensory information. Neuron 92, 1122-1134 (2016).

[30] Sergent, C., Baillet, S. \& Dehaene, S. Timing of the brain events underlying access to consciousness during the attentional blink. Nature neuroscience 8, 1391 (2005).

[31] Van Vugt, B. et al. The threshold for conscious report: Signal loss and response bias in visual and frontal cortex. Science $\mathbf{3 6 0}$, 537-542 (2018).

[32] Cohen, L. et al. The visual word form area: spatial and temporal characterization of an initial stage of reading in normal subjects and posterior split-brain patients. Brain 123, 291-307 (2000).

[33] Lamme, V. A. Why visual attention and awareness are different. Trends in cognitive sciences 7, 12-18 (2003).

[34] Jones, E., Oliphant, T., Peterson, P. et al. SciPy: Open source scientific tools for Python (2001-). URL http://www . scipy .org/. [Online; accessed ;today $i]$.

[35] Kleiner, M. et al. What's new in psychtoolbox-3. Perception 36, 1 (2007).

[36] Pedregosa, F. et al. Scikit-learn: Machine learning in python. Journal of machine learning research 12, 2825-2830 (2011). 